\title{
Compact long-wavelength band Brillouin-erbium fiber laser in a Fabry-Perot resonator
}

\begin{abstract}
We demonstrate compact architecture of multiwavelength L-band Brillouin-Erbium fiber laser in a Fabry-Perot resonator. The laser architecture utilizes a double-pass Brillouin pump pre-amplification technique to increase the Brillouin gain efficiency. The proposed fiber laser structure is able to operate at shot length of single mode fiber and is able to produce high number of output channels with high peak output power. We are able to generate up to 13 and 25 output channels with a constant wavelength separation of $0.089 \mathrm{~nm}$ in a short single mode fiber of $0.1 \mathrm{~km}$ and $0.5 \mathrm{~km}$ respectively.
\end{abstract}

Keyword: Fiber lasers; Brillouin scattering; Erbium; Nonlinear optic 